\title{
Diagnosing social anxiety in Parkinson's disease: characteristics and frequencies according to two diagnostic criteria
}

\author{
Tais S. Moriyama1,2, Marcos Hortes N. Chagas33,4, Laura Silveira-Moriyama5, Vitor Tumas³, Andrew J. Lees5, \\ Jose Alexandre CRIPPA3, Rodrigo A. Bressan 1,2 \\ 1 Hospital Israelita Albert Einstein (HIAE), Instituto Israelita de Ensino e Pesquisa Albert Einstein, São Paulo, SP, Brazil. \\ 2 Universidade Federal de São Paulo (USP). Departamento de Psiquiatria, Laboratório Interdisciplinar de Neurociências Clínicas, São Paulo, SP Brazil. \\ ${ }_{3}^{3}$ Faculdade de Medicina de Ribeirão Preto, Universidade de São Paulo (FMRP-USP), Departamento de Neurociências e Ciências do Comportamento, Ribeirão Preto, SP, Brazil. \\ 4 Universidade Federal de São Carlos (UFSCar), Departamento de Gerontologia, Centro de Ciências Biológicas e da Saúde, São Carlos, SP, Brazil. \\ 5 UCL Institute of Neurology, Reta Lila Weston Institute of Neurological Studies, London, UK.
}

Institution where the study was conducted: Universidade de São Paulo, Departamento de Neurociências e Ciências do Comportamento, Faculdade de Medicina de Ribeirão Preto, Hospital das Clínicas de Ribeirão Preto.

Received: 4/1/2016 - Accepted: 10/28/2016

DOl: 10.1590/0101-60830000000100

\begin{abstract}
Background: Studies found inconsistent frequencies of social anxiety disorder (SAD) in Parkinson's disease (PD) (9.7\%-50\%). Previous reports did not test the impact of applying DSM-IV restrictive criteria that recommends the exclusion of secondary cases when diagnosing SAD in PD. Objective: Our aim is to estimate the frequency of social anxiety according to DSM-IV criteria and according to an inclusive broader approach. Methods: One hundred and ten PD patients were assessed for the presence of SAD using SCID-I, diagnosis of social anxiety were determined according to two different criteria: following and not following DSM-IV recommendation for exclusion of cases though to be secondary to a general medical condition. Results: SAD was present in 34 ( $31 \%$ ) of patients, but 17 (15.5\%) were secondary to a general medical condition. Patients with SAD were significantly younger, had earlier disease onset, had more severe PD symptoms, and were more frequently depressed. There was no difference in demographic and clinical features between primary and secondary SAD. Discussion: We conclude that the use of different diagnostic criteria may have a massive impact in the estimation of frequency of SAD in PD.
\end{abstract}

Moriyama TS et al. / Arch Clin Psychiatry. 2016;43(6):139-42

Keywords: Social anxiety, Parkinson's disease, diagnosis.

\section{Introduction}

Social anxiety disorder (SAD) is characterized by an abnormal fear and avoidance of scrutiny by others (DSM-IV) ${ }^{1}$. It has a pointprevalence of $7.1 \%$, and a life-time prevalence of up to $12.1 \%{ }^{2}$. Anxiety disorders are known to be more prevalent in patients with Parkinson's disease (PD) than in age and gender matched controls with similar debilitating diseases ${ }^{3}$. Despite the fact that anxiety has been largely studied in PD, only a few studies specifically addressed $\mathrm{SAD}$ in PD reporting widely variable prevalence of $9.7 \%^{4}, 11.5 \%{ }^{5}$, $34 \%^{6}$ and $50 \%{ }^{7}$.

One reason for this discrepancy is related to the lack on consensus on how to diagnose SAD in PD patients. DSM IV-TR recommends the exclusion of psychiatric symptoms that are likely attributable to a medical condition ${ }^{1}$ but it is possible that many clinically relevant cases are missed if the DSM-IV criteria is employed. Researchers who follow this recommendation often point out a lower frequencies of SAD in PD than researchers who does not $\left(9.7 \%^{4}, 11.5 \%^{5}\right.$ and $17 \%^{6}$ versus $34 \%^{6}$ and $50 \%^{7}$ respectively), nevertheless, except for a small study including only 24 patients $^{6}$, no work to date has directly compared the frequency of SAD in PD according to the two different criteria in the same PD sample. Although using DSM-IV recommendation may lead to underdiagnosis ignoring such criteria may result in misdiagnosis. Some PD symptoms overlap with anxiety symptoms (like tremor and dysautonomia) and the motor symptoms of PD (which include tremor and difficulty to perform motor tasks appropriately) cause worry and embarrassment, and many times withdraw from social situations. According to DSM-IV diagnosis of primary SAD should be made only for patients presenting all symptoms of social anxiety (criteria A to F) and that are not excluded by the $\mathrm{G}$ and $\mathrm{H}$ criteria.
Patients whose phobic symptoms started close after a disease onset (criterion G) and those that fears or avoids situations that can be related to the presence of a physical condition (criterion $\mathrm{H}$ ) should be considered secondary cases of SAD.

We studied a sample of $110 \mathrm{PD}$ patients aiming to compare the frequency of SAD in PD according to the two different criteria (using DSM-IV exclusion criteria and ignoring it) and to compare the clinical differences between primary and secondary SAD. We also tested the psychometric properties of a short screening instrument for SAD in PD.

\section{Methods}

\section{Study design}

A cross-sectional descriptive study of a clinical sample of patients with PD was performed in a tertiary referral center in Brazil.

\section{Subjects}

One hundred and ten PD patients who fulfilled Queen Square Brain Bank diagnostic criteria ${ }^{8}$ were screened for the presence of SAD. Patients were recruited from Movement Disorders Clinic of the Ribeirão Preto School of Medicine, Brazil. PD patients with dementia were not invited to participate. The presence of dementia was determined by direct interview with the patient and caregiver according to the DSM-IV diagnostic criteria.

The local ethics committees of the three institutions involved in the study approved this study and all patients provided informed consent. 


\section{Clinical evaluation}

An experienced psychiatrist (MHNC) conducted the psychiatric evaluation using a structured clinical interview (SCID-I) ${ }^{9}$ to diagnose current prevalence of SAD according to DSM IV-TR criteria. To fulfill criteria for SAD patients needed to present (1) persistent fear of one or more social or performance situations; (2) exposure to the feared situation almost invariably provokes anxiety; (3) the person recognizes that this fear is unreasonable or excessive; (4) the feared situations are avoided or endured with intense anxiety and distress; (5) symptoms interfere significantly with the person's life or there is marked distress. All participants fulfilling such criteria were coded as SAD cases. Then SAD cases were coded in primary or secondary based on criteria $G$ and $H$ of the SCID. Following strictly DSM-IV criteria the diagnosis of primary SAD was made only in patients fulfilling $\mathrm{G}$ and $\mathrm{H}$ criteria. All other SAD cases were considered secondary SAD.

Participants were also assessed using the previously validated Brazilian version of the Mini Social Phobia Inventory (Mini-SPIN), which is a self-report instrument to measure social anxiety symptoms ${ }^{10}$. Mini-SPIN is a very brief and easy to use instrument, it comprises only 3 items derived from the Social Phobia Inventory. The respondent is asked to rate from 0 to 4 depending on how much they disagree or agree with the following 3 sentences: "Fear of embarrassment causes me to avoid doing things or speaking to people"; "I avoid activities in which I am the center of attention"; "Being embarrassed or looking stupid are among my worse fears". The total score is the sum of scores in each of the three items, varying from 0 to 12 .

The presence of depressive symptoms was characterized using the 15-item shortened version of the Geriatric Depression Scale (GDS15) previously validated in Brazil11.

Clinical data including disease duration, $\mathrm{PD}$ treatment and $\mathrm{PD}$ severity according to the Hoehn and Yahr scale (H\&Y) ${ }^{12}$, the Schwab and England scale $(\mathrm{S} \& \mathrm{E})^{13}$, and a shortened version of the Unified Parkinson's Disease Rating Scale (UPRDS) previously validated in Brazil ${ }^{14}$ was collected on the same day by a movement disorder specialist.

\section{Statistical analysis}

Frequency of SAD (overall, primary and secondary) was calculated. Student $t$-test, Mann Whitney, chi-square and Fisher's exact tests were used to compare the continuous and nominal variables between patients without SAD versus SAD, and also between patients with primary SAD versus secondary SAD.

Receiver Operating Characteristic (ROC) curves were used to determine the best cut-off points in the Mini-SPIN to discriminate between subjects with and without SAD according to SCID-I.

Analysis were performed using the Statistical Package for Social Sciences (SPSS for Mac OS X, version 17.0). Normality requirements for data distribution were confirmed using Shapiro-Wilk test. A significance level of 0.05 was used through.

\section{Results}

Thirty-four (31\%) of the $110 \mathrm{PD}$ patients fulfilled criteria for SAD. Half fulfilled criteria for primary SAD $(\mathrm{N}=17,15.5 \%)$ and the other half fulfilled criteria for secondary SAD. A comparison of clinical data of patients without SAD and with SAD and between primary and secondary SAD is presented in Table 1. Patients with SAD were significantly younger, more frequently in a stable relationship, had significantly younger age at PD onset, had worse motor symptoms, more depressive symptoms and were more frequently depressed. Mini-SPIN score was higher for patients with SAD, as expected. No significant differences were found among primary and secondary SAD.

A ROC curve analysis of Mini-SPIN using diagnosis of SAD according to SCID-I as gold-standard suggested that a cutoff score of $4 / 5$ provided the best balance between sensitivity and specificity. This cutoff score provided a sensitivity of $78.8 \%$, a specificity of $70.6 \%$, a positive predictive value of $56.5 \%$, and a negative predictive value of $87.2 \%$, with an area under the curve of 0.829 .

\section{Discussion}

It is regularly recommended not to exclude secondary cases of mental disorders in PD. The impact of such recommendation in clinical practice has been largely studied for depression ${ }^{15}$ but few data exists to support the extension of this recommendation for SAD in PD. We found SAD to be more common in PD than what is expected in the general population ${ }^{2}$ when using either the stricter or the more general criteria. The frequency of strictly diagnosed primary SAD was $(15.5 \%)$, which is twice higher than that found

Table 1. Demographic and clinical characteristics of Parkinson's Disease (PD) patients evaluated for the presence of SAD

\begin{tabular}{|c|c|c|c|c|c|c|}
\hline & $\begin{array}{l}\text { Without SAD } \\
\text { (WS) }\end{array}$ & SAD & \begin{tabular}{|c|}
$p$ values (WS vs \\
SAD)
\end{tabular} & $\begin{array}{c}\text { Primary SAD } \\
\left(S A D_{1}\right)\end{array}$ & $\begin{array}{c}\text { Secondary SAD } \\
\left(\mathrm{SAD}_{2}\right)\end{array}$ & $\begin{array}{c}\text { p values }\left(S A D_{1}\right. \\
\left.\text { vs } S A D_{2}\right)\end{array}$ \\
\hline Number (\%) of patients & $76(69.0 \%)$ & $34(31 \%)$ & & $17(15.5 \%)$ & $17(15.5 \%)$ & \\
\hline Gender $\%$ of female & $42(55.3 \%)$ & $16(47 \%)$ & $p=0.43$ & $9(52.9 \%)$ & $7(41.17 \%)$ & $p=0.49$ \\
\hline Age (years), mean \pm SD & $63.8 \pm 11.3$ & $55 \pm 13.5$ & $p=0.001^{*}$ & $56.2 \pm 16.1$ & $53.8 \pm 10.5$ & $p=0.61$ \\
\hline Marital status, $\mathrm{N}(\%)$ of married or in a stable relationship & $48(63.2 \%)$ & $28(82.4 \%)$ & $p=0.04^{*}$ & $14(82.4 \%)$ & $14(82.4 \%)$ & $p>0.999$ \\
\hline Employment status, $\mathrm{N}(\%)$ with paid (formal or informal) work & $16(21.1 \%)$ & $10(29.4 \%)$ & $p=0.34$ & $4(23.5 \%)$ & $6(35.3 \%)$ & $p=0.45$ \\
\hline Years of schooling, mean \pm SD & $5.8 \pm 4.7$ & $5.4 \pm 3.1$ & $p=0.66$ & $4.7 \pm 3.2$ & $6.2 \pm 2.9$ & $p=0.12$ \\
\hline Disease duration (years), mean $\pm S D$ & $7.8 \pm 5.5$ & $7.4 \pm 3.9$ & $p=0.75$ & $6.2 \pm 3.5$ & $8.6 \pm 3.9$ & $p=0.11$ \\
\hline Age of disease onset (years), mean \pm SD & $56.0 \pm 12.6$ & $48 \pm 13.5$ & $p=0.002^{*}$ & $49.9 \pm 16.6$ & $45.2 \pm 9.4$ & $p=0.62$ \\
\hline $\begin{array}{l}\text { Most affected side, N (\%) } \\
\text { Right } \\
\text { Left } \\
\text { Both }\end{array}$ & $\begin{array}{l}31(40.7 \%) \\
28(36.8 \%) \\
17(22.3 \%)\end{array}$ & $\begin{array}{c}13(38.2 \%) \\
13(38.2 \%) \\
8(23.5 \%)\end{array}$ & $p=0.97$ & $\begin{array}{l}9(52.9 \%) \\
3(17.6 \%) \\
5(29.4 \%)\end{array}$ & $\begin{array}{c}4(23.5 \%) \\
10(58.8 \%) \\
3(17.6 \%)\end{array}$ & $p=0.07$ \\
\hline Scores on the UPDRS modified motor scale, mean \pm SD & $8.3 \pm 5.4$ & $12.5 \pm 7.3$ & $p=0.003^{*}$ & $10.7 \pm 6.7$ & $14.2 \pm 7.6$ & $p=0.16$ \\
\hline Schwab and England, mean \pm SD & $81.5 \pm 14.6$ & $78 \pm 16.7$ & $p=0.32$ & $79.4 \pm 17.3$ & $76.4 \pm 16.5$ & $p=0.48$ \\
\hline Hoehn and Yahr, mean \pm SD & $2.0 \pm 1.0$ & $2.2 \pm 1.1$ & $p=0.53$ & $2.1 \pm 1.1$ & $2.5 \pm 1.2$ & $p=0.39$ \\
\hline Presence of motor fluctuation, $\mathrm{N}(\%)$ & $21(27.6 \%)$ & $6(17.6 \%)$ & $p=0.34$ & $4(23.5 \%)$ & $2(11.7 \%)$ & $p=0.65$ \\
\hline GDS15 total score, mean \pm SD & $5.0 \pm 3.6$ & $8.0 \pm 3.8$ & $p<0.0005^{*}$ & $7.6 \pm 3.5$ & $6.6 \pm 2.6$ & $p=0.85$ \\
\hline Current depressive episode, N (\%) & $10(13.2 \%)$ & $18(52.9 \%)$ & $p<0.0005^{*}$ & $8(47.1 \%)$ & $10(58.8 \%)$ & $p=0.49$ \\
\hline Mini SPIN score, mean \pm SD & $3.0 \pm 2.8$ & $7.1 \pm 3.1$ & $p<0.0005^{*}$ & $7.6 \pm 3.5$ & $6.6 \pm 2.6$ & $p=0.32$ \\
\hline
\end{tabular}

SAD: Social Anxiety Disorder; UPDRS: Unified Parkinson's Disease Rating Scale; GDS15: 15 items Geriatric Depression Scale; mini SPIN: mini Social Phobia Inventory; BSPS: Brief Social Phobia Scale; SD: standard deviation; *: significant difference $(p<0.05)$. 
in the general population $(7.1 \%)^{2}$. Primary SAD accounted for half of the patients with SAD (17 out of 34), which is consistent with a previous small study in which 7 out of 24 PD patients presented SAD (29.2\%), of whom 4 (16.6\%) had primary SAD. Although our data is consistent with previous works it is prudent to consider that we used a sample of PD patients from a tertiary referral center and that may not be representative of the overall $\mathrm{PD}$ population.

Considering the high frequencies of SAD reported and the burden of this condition for patients and careers, a more targeted and systematic screening should be routinely made in clinical practice. Despite being one of the commonest causes of psychological morbidity in the general population SAD frequently goes unrecognized and undertreated ${ }^{16}$. Data from the Brazilian population indicates that although SAD is present in $10.2 \%$ of university students only $0.8 \%(n=2$ out of 235$)$ had ever been diagnosed or used medication to treat it ${ }^{17}$. A possible approach for increasing the detection of SAD among PD patients is the broad use of self-applicable screening instruments; this can possibly optimize diagnosis without increasing medical appointments length and with no need for referencing patients to psychiatric evaluation. In our sample, using a cut-off score of $4 / 5$, mini-SPIN showed reasonable psychometric properties for the screening of SAD in PD. This scale is realistic within routine clinical setting and could help point-out patients who are suitable for interventions to treat SAD.

The higher frequency of SAD among PD patients may be explained by psychosocial ${ }^{18}$ and neurobiological mechanisms ${ }^{19}$. SAD may be a psychosocial reaction to the stigmatizing PD symptoms such as tremor and bradykinesia, but abnormal function of the striatum has been pointed as one of the key pathophysiological mechanism implicated in $\mathrm{SAD}^{20-23}$ and it is also plausible that the profound disruption of the main catecholaminergic and serotonergic pathways that occurs in $\mathrm{PD}^{24}$ predispose patients to SAD. In our sample, SAD was associated with the intensity of motor symptoms, younger age and with earlier age at disease onset, all of which imply both in higher psychosocial stress and in more severe nigrostriatal dopaminergic denervation 25,26 .

Differentiating primary from secondary SAD might be important for clinical practice. It is plausible that primary SAD should be benefit from standard treatment for SAD in general population, while patients with secondary SAD may benefit from specific treatment strategies focused on PD symptoms like optimization of antiparkinsonian medications and psychological interventions to help patients to cope with PD symptoms with less distress and anti-stigma intervention.

Another issue to be addressed on the association of SAD and PD is depression, which is often a comorbidity with $\mathrm{PD}^{27,28}$. Depression is known to increase the risk of SAD in general populations and one possible explanation for the higher prevalence of anxiety in PD is the presence of other mental disorders (such as depression and dementia) that might hamper anxiety symptoms evaluation ${ }^{29}$ or predispose the patients to SAD. The co-occurrence of anxiety and depression is known to be more common in PD patients than in healthy controls or in the general population ${ }^{30}$. In our sample the PD patients with SAD had higher scores in the GDS15 scale and were more likely to be depressed. Due to the cross-sectional nature of our study it is not possible to explore the nature of the association between SAD and depression.

\section{Conclusion}

We conclude that the use of different diagnostic criteria may have a massive impact in the estimation of frequency of SAD in PD, besides we do not find differences between secondary and primary $\mathrm{SAD}$. We also suggest that quick screening questionnaires might help guide clinicians in the diagnosis of SAD.

\section{Conflicts of interest}

None.

\section{Acknowledgements}

Tais S. Moriyama received financial support from Instituto Uniemp - Hospital Israelita Albert Einstein. Rodrigo A. Bressan received financial support from Instituto Uniemp - Hospital Israelita Albert Einstein and is recipient of a Conselho Nacional de Desenvolvimento Científico e Tecnológico (CNPq, Brazil) fellowships award. José Alexandre Crippa is recipient of a Conselho Nacional de Desenvolvimento Científico e Tecnológico (CNPq, Brazil) fellowships award. Laura Silveira-Moriyama was a beneficiary of a Reta Lila Weston fellowship.

\section{References}

1. American Psychiatric Association. Social Phobia (Social Anxiety Disorder). In: APA, ed. Diagnostic and Statistical Manual of Mental Disorders, Fourth Edition, Text Revision ed. Washington, DC: American Psychiatric Association; 2000. p. 450-6.

2. Stein MB, Stein DJ. Social anxiety disorder. Lancet. 2008;371:1115-25.

3. Menza MA, Mark MH. Parkinson's disease and depression: the relationship to disability and personality. J Neuropsychiatry Clin Neurosci. 1994;6:165-9.

4. Pontone GM, Williams JR, Anderson KE, Chase G, Goldstein SA, Grill $S$, et al. Prevalence of anxiety disorders and anxiety subtypes in patients with Parkinson's disease. Mov Disord. 2009;24:1333-8.

5. de Rijk C, Bijl RV. [Prevalence of mental disorders in persons with Parkinson's disease]. Ned Tijdschr Geneeskd. 1998;142:27-31.

6. Stein MB, Heuser IJ, Juncos JL, Uhde TW. Anxiety disorders in patients with Parkinson's disease. Am J Psychiatry. 1990;147:217-20.

7. Kummer A, Cardoso F, Teixeira AL. Frequency of social phobia and psychometric properties of the Liebowitz social anxiety scale in Parkinson's disease. Mov Disord. 2008;23:1739-43.

8. Gibb WR, Lees AJ. The relevance of the Lewy body to the pathogenesis of idiopathic Parkinson's disease. J Neurol Neurosurg Psychiatry. 1988;51:745-52.

9. Del-Ben CM, Vilela JAA, Crippa JAS, Hallak JEC, Labate CM, Zuardi AW. Confiability of the Structured Clinical Interview for DSM-IV - Clinical Version translated into Portuguese. Rev Bras Psiquiatr. 2001:156-9.

10. de Lima Osorio F, Crippa JA, Loureiro SR. A study of the discriminative validity of a screening tool (MINI-SPIN) for social anxiety disorder applied to Brazilian university students. Eur Psychiatry. 2007;22:239-43.

11. Tumas V, Rodrigues GG, Farias TL, Crippa JA. The accuracy of diagnosis of major depression in patients with Parkinson's disease: a comparative study among the UPDRS, the geriatric depression scale and the Beck depression inventory. Arq Neuropsiquiatr. 2008;66:152-6.

12. Hoehn MM, Yahr MD. Parkinsonism: onset, progression and mortality. Neurology. 1967;17:427-42.

13. Schwab RS, England AC. Projection technique for evaluating surgery in Parkinson's disease. Edinburgh: Livingstone; 1969.

14. Tumas V, Ujikawa LT, Ferreira GM. Utility and reliability of a simplified clinical scale for Parkinson's disease. Arq Neuropsiquiatr. 2004;62:220-1.

15. Marsh L, McDonald WM, Cummings J, Ravina B. Provisional diagnostic criteria for depression in Parkinson's disease: report of an NINDS/NIMH Work Group. Mov Disord. 2006;21:148-58.

16. Kessler RC. The impairments caused by social phobia in the general population: implications for intervention. Acta Psychiatr Scand Suppl. 2003:19-27.

17. Crippa JA, Loureiro SR, Baptista CA, Osorio F. Are there differences between early- and late-onset social anxiety disorder? Rev Bras Psiquiatr. 2007;29:195-6; author reply 196-7.

18. Evans D, Norman P. Illness representations, coping and psychological adjustment to Parkinson's disease. Psychol Health. 2009;24:1181-96.

19. Robinson HM, Hood SD, Bell CJ, Nutt DJ. Dopamine and social anxiety disorder. Rev Bras Psiquiatr. 2006;28:263-4.

20. Schneier FR, Liebowitz MR, Abi-Dargham A, Zea-Ponce Y, Lin SH, Laruelle M. Low dopamine $\mathrm{D}(2)$ receptor binding potential in social phobia. Am J Psychiatry. 2000;157:457-9.

21. Tiihonen J, Kuikka J, Bergstrom K, Lepola U, Koponen H, Leinonen E. Dopamine reuptake site densities in patients with social phobia. Am J Psychiatry. 1997;154:239-42.

22. Schneier FR, Martinez D, Abi-Dargham A, Zea-Ponce Y, Simpson HB, Liebowitz MR, et al. Striatal dopamine D(2) receptor availability in OCD 
with and without comorbid social anxiety disorder: preliminary findings. Depress Anxiety. 2008;25:1-7.

23. van der Wee NJ, van Veen JF, Stevens H, van Vliet IM, van Rijk PP, Westenberg HG. Increased serotonin and dopamine transporter binding in psychotropic medication-naive patients with generalized social anxiety disorder shown by 123I-beta-(4-iodophenyl)-tropane SPECT. J Nucl Med. 2008;49:757-63.

24. Braak H, Del Tredici K, Rub U, de Vos RA, Jansen Steur EN, Braak E. Staging of brain pathology related to sporadic Parkinson's disease. Neurobiol Aging. 2003;24:197-211.

25. Felicio AC, Shih MC, Godeiro-Junior C, Andrade LA, Bressan RA, Ferraz HB. Molecular imaging studies in Parkinson disease: reducing diagnostic uncertainty. Neurologist. 2009;15:6-16.

26. Shih MC, Franco de Andrade LA, Amaro E Jr, Felicio AC, Ferraz HB, Wagner J, et al. Higher nigrostriatal dopamine neuron loss in early than late onset Parkinson's disease?--a [99mTc]-TRODAT-1 SPECT study. Mov Disord. 2007;22:863-6.

27. McDonald WM, Richard IH, DeLong MR. Prevalence, etiology, and treatment of depression in Parkinson's disease. Biol Psychiatry. 2003;54:363-75.

28. Nakabayashi TIK, Chagas MHN, Corrêa ACL, Tumas V, Loureiro SR, Crippa JA. Prevalence of depression in Parkinson's disease. Rev Psiquiatr Clín. 2008;35:219-27.

29. Nuti A, Ceravolo R, Piccinni A, Dell'Agnello G, Bellini G, Gambaccini $\mathrm{G}$, et al. Psychiatric comorbidity in a population of Parkinson's disease patients. Eur J Neurol. 2004;11:315-20.

30. Menza MA, Robertson-Hoffman DE, Bonapace AS. Parkinson's disease and anxiety: comorbidity with depression. Biol Psychiatry. 1993;34 465-70. 